\title{
A REMARK ON SETS IN INFINITE DIMENSIONAL SPACES WITH FULL OR ZERO CAPACITY
}

\author{
JIAGANG REN \\ SCHOOL OF MATHEMATICS AND COMPUTATIONAL SCIENCE \\ ZHONGSHAN UNIVERSITY \\ GUANGZHOU, GUANGDONG 510275 \\ P.R.CHINA \\ MICHAEL RÖCKNER \\ FAKULTÄT FÜR MATHEMATIK \\ UNIVERSITÄT BIELEFELD \\ 33615 BIELEFELD \\ GERMANY
}

\begin{abstract}
We give a simple proof that for classical Dirichlet forms on infinite dimensional linear state spaces the intrinsic closure of a set of full measure has full capacity. Furthermore, we show that the $C_{1, q}$-capacity of a set, enlarged by adding the linear span of a basis in the generalized CameronMartin space remains zero if it was zero for slightly bigger capacities a priori.
\end{abstract}

\section{INTRODUCTION, FRAMEWORK AND A RESULT ON SETS WITH FULL CAPACITY}

In infinite dimensional analysis the question whether a given set has zero or full capacity (in the sense that its compliment has zero capacity) is much less studied than in finite dimensions. This question is of importance, since roughly speaking capacity zero sets are not hit by the underlying process whereas a set of full capacity carries the process for all times. The first aim of this paper is to give a simple analytic proof for the fact that the intrinsic closure of a set of full measure has full capacity (cf. Theorem 1.4 below). This fact is essentially known to experts. We refer e.g. to [7] where this result was proved for a class of Dirichlet forms with non-flat underlying state space. But there is no reference for this result for general classical Dirichlet forms of gradient type on linear state spaces. In this case there is quite an easy proof which we present below. The second aim of this paper is to prove a result one would expect, but appears to be new. Namely, we prove that the $C_{1, q}$-capacity of a set, enlarged by adding all finite linear combinations of a basis in the generalized Cameron-Martin space, remains zero if it was zero for (slightly bigger) $C_{r, p}$-capacities, $r>1, p>q$, a priori (cf. Theorem 3.3 below). Let us first describe our framework, in which we strictly follow [2].

Let $E$ be a separable Banach space over $\mathbb{R}$. Let $E^{\prime}$ denote its dual and $\mathcal{B}(E)$ its Borel $\sigma$-algebra. Let $(H,\langle\rangle$,$) be a Hilbert space such that H \subset E$ continuously and densely. 
Identifying $H$ with its dual $H^{\prime}$ by Riesz's isomorphism, we have

$$
E^{\prime} \subset H \subset E
$$

where both embeddings are continuous and dense. In particular, it follows for the dualization $E^{\prime}\langle,\rangle_{E}: E^{\prime} \times E \rightarrow \mathbb{R}$ that

$$
{ }_{E^{\prime}}\langle l, h\rangle_{E}=\langle l, h\rangle_{H} \text { for all } l \in E^{\prime}, h \in H .
$$

Furthermore

$$
H=\left\{z \in E \mid \sup \left\{{ }_{E^{\prime}}\langle l, z\rangle_{E} \mid l \in E^{\prime} \text { with }\|l\|_{H} \leqslant 1\right\}<\infty\right\} .
$$

The norm in $E$ and $H$ we denote by \|\|$_{E}$ and \|\|$_{H}$ respectively.

Let

$$
\mathcal{F} C_{b}^{\infty}:=\left\{g\left(l_{1}, \cdots, l_{N}\right) \mid N \in \mathbb{N}, g \in C_{b}^{\infty}\left(\mathbb{R}^{N}\right), l_{1}, \cdots, l_{N} \in E^{\prime}\right\},
$$

where $C_{b}^{\infty}\left(\mathbb{R}^{N}\right)$ denotes the set of all infinitely differentiable bounded functions with all derivatives bounded. For $u \in \mathcal{F} C_{b}^{\infty}\left(\mathbb{R}^{N}\right)$ and $z \in E$ we define $\nabla u(z) \in H$ by

$$
\langle\nabla u(z), h\rangle_{H}=\frac{\partial u}{\partial h}(z):=\left.\frac{d}{d t} u(z+t h)\right|_{t=0} .
$$

Let $\mu$ be a probability measure on $(E, \mathcal{B}(E))$ and denote the corresponding real $L^{p_{-}}$ spaces by $L^{p}(E, \mu), p \in[1, \infty]$, and define

$$
\mathcal{E}_{\mu}(u, v):=\int_{E}\langle\nabla u(z), \nabla v(z)\rangle_{H} \mu(d z) ; u, v \in \mathcal{F} C_{b}^{\infty} .
$$

For a set $\mathcal{D}$ of $\mathcal{B}(E)$-measurable functions on $E$ we denote the corresponding $\mu$-classes by $\widetilde{\mathcal{D}}^{\mu}$. Throughout this paper we assume that the following hypothesis is fulfilled

(H1) If $u \in \mathcal{F} C_{b}^{\infty}$ such that $u=0 \mu$-a.e., then $\nabla u=0 \mu$-a.e. and the (thus on $L^{2}(E, \mu)$ well-defined) positive definite symmetric bilinear form $\left(\mathcal{E}_{\mu},{\widetilde{\mathcal{F} C_{b}^{\infty}}}^{\mu}\right)$ is closable on $L^{2}(E, \mu)$.

Under condition (H1) the Hilbert space $H$ is sometimes called generalized CameronMartin space of $\mu$. We refer e.g. to [5] for the definition of closability and denote the closure of $\left(\mathcal{E}_{\mu},{\widehat{\mathcal{F} C_{b}^{\infty}}}^{\mu}\right)$ on $L^{2}(E, \mu)$ by $\left(\mathcal{E}_{\mu}, H_{0}^{1,2}(E, \mu)\right)$. Then $\left(\mathcal{E}_{\mu}, H_{0}^{1,2}(E, \mu)\right)$ is a symmetric Dirichlet form (see e.g. [5]).

Remark 1.1 (i) For sufficient conditions for (H1) we refer to [2]. We note that those conditions are also necessary, if one requires all partial derivatives to be closable separately (see [2] for details).

(ii) Closability of the form $\left(\mathcal{E}_{\mu},{\widetilde{\mathcal{F} C_{b}^{\infty}}}^{\mu}\right)$ on $L^{2}(E, \mu)$ is equivalent to the closability of the operator

$$
\nabla:{\widetilde{\mathcal{F} C_{b}^{\infty}}}^{\mu} \subset L^{2}(E, \mu) \longrightarrow L^{2}(E \rightarrow H, \mu) .
$$

We denote its closure (whose domain is, of course, $H_{0}^{1,2}(E, \mu)$ ) again by $\nabla$.

If this operator is closable, then also for $p \geqslant 2$

$$
\nabla:{\widetilde{\mathcal{F} C_{b}^{\infty}}}^{\mu} \subset L^{p}(E, \mu) \longrightarrow L^{p}(E \rightarrow H, \mu)
$$


is closable. Indeed, if $u_{n} \rightarrow 0$ in $L^{p}(E, \mu)$ as $n \rightarrow \infty$ and $\left(\nabla u_{n}\right)_{n \in \mathbb{N}}$ is a Cauchy sequence in $L^{p}(E \rightarrow H, \mu)$, then the same holds in $L^{2}(E, \mu)$ and $L^{2}(E \rightarrow H, \mu)$ respectively. By assumption it follows that $\nabla u_{n} \rightarrow 0$ in $L^{2}(E \rightarrow H, \mu)$ as $n \rightarrow \infty$, hence in $\mu$-measure, so by Fatou's Lemma

$$
\int\left\|\nabla u_{n}\right\|_{H}^{p} d \mu \leqslant \liminf _{m \rightarrow \infty} \int\left\|\nabla u_{n}-\nabla u_{m}\right\|_{H}^{p} d \mu .
$$

But the right hand side can be made arbitrarily small.

(iii) Assuming that for $p \geqslant 1$

$$
\nabla:{\widetilde{\mathcal{F} C_{b}^{\infty}}}^{\mu} \subset L^{p}(E, \mu) \longrightarrow L^{p}(E \rightarrow H, \mu) \text { is closable, }
$$

we can prove all what follows for $p \geqslant 1$ instead of $p=2$ with entirely similar proofs. For simplicity we restrict, however, to the case $p=2$. The definition of capacities, however, we give for all $p \geqslant 1$ below.

(iv) We refer to [2] and [1] for examples for $\mu$ satisfying (H1). These examples include the white noise measure on $E$, i.e. the centered Gaussian measure on $(E, \mathcal{B}(E))$ with Cameron-Martin space $H$. But many other Gaussian measures and moreover Gibbs measures from statistical mechanics are included.

If for $p \in[1, \infty)$ condition (1.7) holds, we denote the closure by $\left(\nabla, H_{0}^{1, p}(E, \mu)\right)$. For notational convenience we then set as usual for $p \geqslant 1$

$$
\|u\|_{1, p}:=\left(\int\left(\|\nabla u\|_{H}^{p}+|u|^{p}\right) d \mu\right)^{1 / p}, u \in H_{0}^{1, p}(E, \mu) .
$$

Now we recall the definition of capacity and intrinsic metric.

Definition 1.1. (i) For $U \subset E, U$ open, and $p \in[1, \infty)$, we set

$$
C_{1, p}(U):=\inf \left\{\|u\|_{1, p}^{p} \mid u \in H_{0}^{1, p}(E, \mu), u \geqslant 1 \mu \text {-a.e. on } U\right\}
$$

and for arbitrary $A \subset E$

$$
C_{1, p}(A):=\inf \left\{C_{1, p}(U) \mid A \subset U\right\} .
$$

$C_{1, p}(A)$ is called capacity of $A$.

(ii) A function $f: A \mapsto \mathbb{R}, A \subset E$, is called $C_{1, p}$-quasicontinuous if there exist closed sets $A_{n} \subset A, n \in \mathbb{N}$, such that $f \uparrow_{A_{n}}$ is continuous for all $n \in \mathbb{N}$ and $\lim _{n \rightarrow \infty} C_{1, p}\left(E \backslash A_{n}\right)=$ 0 .

Definition 1.2. For $x, y \in E$ set

$$
\rho(x, y):=\sup \left\{f(x)-f(y) \mid f \in \mathcal{F} C_{b}^{\infty} \text { with }\|\nabla f\|_{H} \leqslant 1\right\} .
$$

$\rho$ is called intrinsic metric of $\left(\mathcal{E}_{\mu}, H_{0}^{1,2}(E, \mu)\right)$.

The following is well-known. The proof is easy and included for the reader's convenience.

Lemma 1.3. Let $x, y \in E$. Then

$$
\rho(x, y)=\|x-y\|_{H},
$$

where we set $\|z\|_{H}:=+\infty$ if $z \in E \backslash H$. 
Proof. Let $f \in \mathcal{F} C_{b}^{\infty}$ with $\|\nabla f\|_{H} \leqslant 1$ and assume $x-y \in H$. Then

$$
\begin{aligned}
f(x)-f(y) & =\int_{0}^{1} E_{E^{\prime}}\left\langle D f(y+t(x-y), x-y\rangle_{E} d t\right. \\
& =\int_{0}^{1}\langle\nabla f(y+t(x-y)), x-y\rangle_{H} d t \\
& \leqslant\|x-y\|_{H} .
\end{aligned}
$$

Here $D f$ denotes the Fréchet derivative of $f$. So,

$$
\rho(x, y) \leqslant\|x-y\|_{H} \text { for all } x, y \in E .
$$

Conversely, let $\chi_{n} \in C_{b}^{\infty}(\mathbb{R})$ satisfying $\left|\chi_{n}\right| \leqslant n+1,\left|\chi_{n}^{\prime}\right| \leqslant 1 \mid, \chi_{n}(s)=s$ for all $s \in[-n, n]$. The for $l \in E^{\prime}$ such that $\|l\|_{H} \leqslant 1$ we have $\left\|\nabla \chi_{n}(l)\right\|_{H} \leqslant 1$ for all $n \in \mathbb{N}$, hence for $x, y \in E$

$$
\begin{aligned}
& E^{\prime}\langle l, x-y\rangle_{E} \leqslant \sup _{n}\left(\chi_{n}\left({ }_{E^{\prime}}\langle l, x\rangle_{E}\right)-\chi\left({ }_{E^{\prime}}\langle l, y\rangle_{E}\right)\right) \\
& \quad \leqslant \rho(x, y) .
\end{aligned}
$$

So, by (1.3)

$$
\|x-y\|_{H} \leqslant \rho(x, y) .
$$

For $A \subset E$ as usual we set

$$
\rho_{A}(x):=\inf \{\rho(x, y) \mid y \in A\}, x \in E .
$$

Now we can formulate the main result of this section which we shall prove in the next section.

Theorem 1.4. Assume hypothesis (H1) holds. Let $A \in \mathcal{B}(E)$ such that $\mu(A)=1$. Then $C_{1,2}\left(\rho_{A}>0\right)=0$, i.e., the $\rho$-closure of $A$ has full $C_{1,2}$-capacity.

\section{PROOF OF THEOREM 1.4}

Throughout this section hypothesis (H1) is assumed to hold. Before we can prove Theorem 1.4, we need the following lemma.

Lemma 2.1. Let $K \subset E$ be $\|\cdot\|_{E}$-compact and $c \in(0, \infty)$. Then $\rho_{K}$ is $\mathcal{B}(E)$-measurable and

$$
\rho_{K} \wedge c \in H_{0}^{1,2}(E, \mu) \text { and }\left\|\nabla\left(\rho_{K} \wedge c\right)\right\|_{H} \leqslant 1 .
$$

Furthermore, $\rho_{K} \wedge c$ is $C_{1,2}$-quasicontinuous.

Proof. Let $\left\{e_{i} \mid i \in \mathbb{N}\right\} \subset E^{\prime}$ be an orthonormal basis of $H$ separating the points of $E$, and for $n \in \mathbb{N}$ define $P_{n}: E \mapsto E_{n}:=\operatorname{span}\left\{e_{1}, \cdots, e_{n}\right\}$ by

$$
P_{n} z:=\sum_{i=1}^{n}{ }_{E^{\prime}}\left\langle e_{i}, z\right\rangle_{E} e_{i}, z \in E .
$$

Fix $y \in E$. By a simple approximation argument on $E_{N}$ we see that

$$
u_{n}(x):=\left\|P_{n} x-P_{n} y\right\|_{H} \wedge c, x \in E,
$$

is a function in $H_{0}^{1,2}(E, \mu)$ with $\left\|\nabla u_{n}\right\|_{H} \leqslant 1$. Clearly

$$
u_{n}(x) \uparrow v_{y}(x):=\|x-y\|_{H} \wedge c \text { for all } x \in E .
$$


Hence by [5, Chap. I, Lemma 2.12] for $y \in E, v_{y} \in H_{0}^{1,2}(E, \mu)$ with $\left\|\nabla v_{y}\right\|_{H} \leqslant 1$ and $u_{n} \rightarrow v_{y}$ weakly in $H_{0}^{1,2}(E, \mu)$, hence the Cesaro mean of a subsequence converges strongly in $H_{0}^{1,2}(E, \mu)$. A standard argument of Egorov type for capacities (cf. [5, Chap.III, Sect. 3]) implies that selecting a subsequence if necessary, this Cesaro-mean converges $C_{1,2^{-}}$ quasiuniformly, i.e., uniformly on closed sets whose compliments have arbitrarily small $C_{1,2}$-capacity. Hence by (2.1) $v_{y}$ is $C_{1,2}$ quasicontinuous.

Claim. Let $\operatorname{dim} E<\infty$. Then the assertions of the lemma hold even without assuming $K$ to be compact.

Since $\operatorname{dim} E<\infty$, we have $H=E$ and \|\|$_{H}$ and \|\|$_{E}$ are equivalent norms. Let $\left\{y_{n} \mid n \in \mathbb{N}\right\}$ be a countable $\|\cdot\|_{H^{-}}$dense subset of $K$ and defining

$$
v_{N}:=\inf \left\{v_{y_{1}}, \cdots v_{y_{N}}\right\}, N \in \mathbb{N},
$$

we have

$$
\rho_{K} \wedge c=\inf _{N} v_{N} \quad \text { on } E .
$$

Furthermore, (cf. e.g. [5, Chap. IV, Sect 4]) $v_{N} \in H_{0}^{1}(E, \mu)$ with

$$
\left\|\nabla v_{N}\right\|_{H} \leqslant \sup \left\{\left\|\nabla v_{y_{1}}\right\|_{H}, \cdots,\left\|\nabla v_{y_{N}}\right\|_{H}\right\}
$$

hence

$$
\left\|\nabla v_{N}\right\|_{H} \leqslant 1 \text { for all } N \in \mathbb{N} .
$$

Therefore, the claim follows by the same arguments as above.

Now we go back to the general case. First we show that for all $x \in E$

$$
\rho_{P_{n} K}\left(P_{n} x\right) \uparrow \rho_{K}(x) \text { as } n \rightarrow \infty .
$$

(So, in particular, $\rho_{K}$ is $\mathcal{B}(E)$-measurable.)

Let $x \in E$. Obviously, $\rho_{P_{n} K}\left(P_{n} x\right)$ is increasing with $n$ and

$$
\sup _{n} \rho_{P_{n} K}\left(P_{n} x\right) \leqslant \rho_{K}(x)
$$

(cf. (2.1)). To prove the dual inequality we may assume that $\sup _{n} \rho_{P_{n} K}\left(P_{n} x\right)<\infty$. Let $a \in(0, \infty)$ such that

$$
\sup _{n} \rho_{P_{n} K}\left(P_{n} x\right)<a .
$$

Then there exist $k_{n} \in K$ such that

$$
\left\|P_{n} x-P_{n} k_{n}\right\|_{H}<a \text { for all } n \in \mathbb{N} .
$$

Since balls in $H$ are weakly compact and $K$ is compact in $E$, we can find a subsequence such that $k_{n_{j}} \stackrel{j \rightarrow \infty}{\longrightarrow} k \in K$ w.r.t. \|\|$_{E}$ and $P_{n_{j}} x-P_{n_{j}} k_{n_{j}} \stackrel{j \rightarrow \infty}{\longrightarrow} h \in H$ weakly in $H$. Hence for all $i \in \mathbb{N}$ by $(1.2)$

$$
\begin{aligned}
{ }_{E^{\prime}}\left\langle e_{i}, h\right\rangle_{E} & =\lim _{j \rightarrow \infty}{ }_{E^{\prime}}\left\langle e_{i}, P_{n_{j}} x-P_{n_{j}} k_{n_{j}}\right\rangle_{E} \\
& =\lim _{j \rightarrow \infty}{ }_{E^{\prime}}\left\langle e_{i}, x-k_{n_{j}}\right\rangle_{E} \\
& ={ }_{E^{\prime}}\left\langle e_{i}, x-k\right\rangle_{E} .
\end{aligned}
$$

Since $\left\{e_{i} \mid i \in \mathbb{N}\right\}$ separates the points of $E$, it follows that $x-k=h$ and by (2.3) that

$$
\|x-k\|_{H} \leqslant \liminf _{j \rightarrow \infty}\left\|P_{n_{j}} x-P_{n_{j}} k_{n_{j}}\right\|_{H} \leqslant a .
$$

In particular, $\rho_{K}(x)<\infty$. 
Now suppose that for some $\varepsilon \in\left(0, \rho_{K}(x)\right)$

$$
\sup _{n} \rho_{P_{n} K}\left(P_{n} x\right)<\rho_{K}(x)-\varepsilon .
$$

Then applying the above with $a:=\rho_{K}(x)-\varepsilon$, we get a contradiction from (2.4). So, (2.2) is proved. Now the assertions follow from the claim and the same arguments used for its proof.

Proof of Theorem 1.4. By inner regularity there exist compact sets $K_{n} \subset A, n \in \mathbb{N}$, such that $\mu\left(K_{n}\right) \uparrow \mu(A)=1$. Let

$$
u_{n}:=\rho_{K_{n}} \wedge 1, \quad n \in \mathbb{N} .
$$

Then

$$
u:=\inf _{n} u_{n}=\lim _{n \rightarrow \infty} u_{n} \geqslant \rho_{A} \wedge 1 \quad \text { on } E .
$$

Furthermore, $u=0$ on $\bigcup_{n \geqslant 1} K_{n}$, hence $u=0 \mu$-a.e.

By Lemma 2.1 and the same arguments as in its proof we obtain that $u$ is $C_{1,2}$-quasicontinuous, hence by [5, Chap. III, Proposition 3.9] $C_{1,2}(\{u>0\})=0$. But by (2.5), $\left\{\rho_{A}>0\right\} \subset\{u>0\}$ and the assertion is proved.

\section{A RESULT ON SETS WITH ZERO CAPACITY}

Let $\left\{h_{1}, h_{2}, \cdots\right\}$ be an ONB of $H$ and let $E_{n}$ denote the linear span of $\left\{h_{1}, \cdots, h_{n}\right\}$ and set $K:=\cup_{n} E_{n}$. A sufficient condition on $A$ for $\mu(A+K)=0$ is given in [4]. Now we look for a condition which implies $C_{p, 1}(A+K)=0$. But we have to work under an additional quasi-invariance hypothesis.

For $k \in H$ define

$$
\tau_{k}(z):=z-k, \quad z \in E .
$$

(H2) For all $k \in H, \mu$ is $k$-quasi-invariant, i.e., $\mu \circ \tau_{s k}^{-1} \cong \mu$ for all $s \in \mathbb{R}$, and we assume the Radon-Nikodym derivatives

$$
a_{s k}^{\mu}:=\frac{d\left(\mu \circ \tau_{s k}^{-1}\right)}{d \mu}, s \in \mathbb{R},
$$

to have the following properties:

(H2a) $a_{s k}^{\mu} \in \cap_{q \geqslant 1} L^{q}(E ; \mu)$, for all $s \in \mathbb{R}$, and for all $q \in[1, \infty)$ the function $s \longmapsto\left\|a_{s k}^{\mu}\right\|_{q}$ is locally bounded on $\mathbb{R}$.

(H2b) For all compact $C \subset \mathbb{R}$

$$
\int_{C} \frac{1}{a_{s k}^{\mu}(z)} d s<\infty \text { for } \mu \text {-a.e. } z \in E \text {. }
$$

Here $d s$ denotes Lebesgue measure on $\mathbb{R}$.

Choosing appropriate versions by [1, Prop. 2.4] we may always assume that $a_{s k}^{\mu}(z)$ is jointly measurable in $s$ and $z$ and that $(\mathrm{H} 2 \mathrm{~b})$ holds for all $z \in E$ (rather than only $\mu$-a.e. $z \in E)$.

For examples of measures $\mu$ satisfying condition (H2) we refer to [6, Section 3]. As shown in [2] hypothesis (H2) implies (H1). 
We need Sobolev spaces with differentiability index higher than 1. Analogue to the gradient operator $\nabla$, we define the iterated gradient $\nabla^{2}$ on $\mathcal{F} C_{b}^{\infty}$ by

$$
\left\langle\nabla^{2} u(z), h_{1} \otimes h_{2}\right\rangle_{H \otimes H}:=\left.\frac{d^{2}}{d t d s} u\left(z+t h_{1}+s_{2} h_{2}\right)\right|_{t=0} .
$$

Assume that

$$
\text { (H3) } \nabla^{2}:{\widetilde{\mathcal{F} C_{b}^{\infty}}}^{\mu} \subset L^{p}(E, \mu) \longrightarrow L^{p}(E \rightarrow H \otimes H, \mu) \text { is closable for } p \geqslant 1 .
$$

We define

$$
H_{0}^{2, p}:=\left\{u \in L^{p} \mid\|u\|_{2, p}:=\left(\int\left(\|\nabla u\|_{H^{\otimes} H}^{p}+\|\nabla u\|_{H}^{p}+|u|^{p}\right) d \mu\right)^{1 / p}<\infty\right\}
$$

and the fractional Sobolev spaces $H_{0}^{r, p}(1<p<\infty, 1<r<2)$ are defined by real interpolation as follows.

Definition 3.1. For $1<p<\infty, 1<r<2$ we define

$$
H_{r}^{p}=\left(H_{0}^{1, p}, H_{0}^{2, p}\right)_{r-1, p}
$$

where $(\cdot, \cdot)$ denotes the real interpolation space, see e.g. $[3,9]$.

The norm in $H_{0}^{r, p}$ is given by the discrete K-method:

$$
\|f\|_{r, p}=\left(\sum_{n=1}^{\infty}\left|2^{n(r-1)} K\left(2^{-n}, f\right)\right|^{p}\right)^{1 / p}<\infty .
$$

where

$$
K(\epsilon, f)=\inf \left\{\left\|f_{1}\right\|_{1, p}+\epsilon\left\|f_{2}\right\|_{2, p}, f_{1}+f_{2}=f, f_{1} \in H_{0}^{1, p}, f_{2} \in H_{0}^{2, p}\right\}
$$

It follows by a standard interpolation argument that $H_{0}^{r, p}$ is uniformly convex (see e.g. [4]) and we know from the denseness of $\mathcal{F} C_{b}^{\infty}$ in $H_{0}^{2, p}$ and [3, Th. 3.4.2] that $\mathcal{F} C_{b}^{\infty}$ is dense in $H_{0}^{r, p}$. A combination of these two facts implies that every $u \in H_{0}^{r, p}$ has a $C_{r, p}$-quasicontinuous redefinition which we denote by $\tilde{u}$.

For $1<p<\infty, 1 \leqslant r \leqslant 2$ we define, for a $[0, \infty]$-valued lower semi-continuous functions $h$ on $E$

$$
C_{r, p}(h):=\inf \left\{\|u\|_{r, p}^{p} ; u \in H_{0}^{r, p}, u \geqslant h, \text { a.e. }\right\},
$$

and for an arbitrary $[-\infty, \infty]$-valued function $f$ on $E$

$$
C_{r, p}(f):=\inf \left\{C_{r, p}(h) ; h \text { is l.s.c.and } h(x) \geqslant|f(x)| \forall x\right\} .
$$

This definition is an extension of the previous one for sets in the sense that for any $B \subset E$,

$$
C_{r, p}(B)=C_{r, p}\left(1_{B}\right) .
$$

The following result is parallel to Shigekawa [8] which is stated for Bessel capacities. We omit the proof which is the same as in [8]. Note that (3.8) is implicit in [8].

Theorem 3.2. Fix $q \in(1, \infty), r \in(1,2], \beta \in(0,1]$ and $0 \leqslant \gamma<\beta / q$. Then there exists a constant $C=C(q, r, \beta, \gamma)$ such that If $\xi:[0, T]^{n} \times E \mapsto \mathbb{R},(t, z) \mapsto \xi_{t}(z)$ is measurable and if

$$
A(\xi):=\sup _{s \neq t, s, t \in[0, T]^{n}} \frac{\left\|\xi_{t}-\xi_{s}\right\|_{r, q}^{q}}{|t-s|^{n+\beta}}<\infty
$$


then $\left\{\xi_{t}\right\}$ has a version $\left\{\tilde{\xi}_{t}\right\}$ such that $\tilde{\xi}_{t}$ is $C_{r, q}$-quasi-continuous for every $t \in[0, T]^{n}$ and

$$
C_{r, q}\left(\sup _{s \neq t} \frac{\left|\tilde{\xi}_{t}-\tilde{\xi}_{s}\right|}{|t-s|^{\gamma}}\right) \leqslant C A(\xi) .
$$

Now we state the main result of this section.

Theorem 3.3. Suppose (H2) and (H3) hold and let $A \subset E, p>1$. If for any $n$ there exists a pair $\left(r_{n}, p_{n}\right) \in(1,2] \times(1, p)$ with $r_{n}>n p_{n}^{-1}+1$ such that

$$
C_{r_{n}, p_{n}}(A)=0, \forall n
$$

then $C_{1, p}(A+K)=0$.

Since capacities are continuous from below Theorem 3.3 immediately follows from Proposition 3.5 below. First we need a lemma.

Fix $n \in \mathbb{N}$ and define for $t \in[-M, M]^{n}$ and $f: E \mapsto \mathbb{R}$ a function $f(t):=f(\cdot+$ $\left.\sum_{i=1}^{n} t_{i} h_{i}\right)$.

Lemma 3.4. Fix $n \in \mathbb{N}$ and let $p>q>1$ and $r \in(1,2]$. Then there exists a constant $C:=C(p, q, r, T)$ such that for all $f \in H_{0}^{r, p}$

$$
\|f(t)-f(s)\|_{1, q} \leqslant C\|f\|_{r, p}|t-s|^{r-1}
$$

for all $s, t \in[0, T]^{n}$.

Proof. By the same argument as in [6], we can prove that for any $T>0, p>1, q \in(0, p)$ there exists a constant $C_{1}=C_{1}(p, q, T)$ such that for all $f \in H_{0}^{2, p}$ and $(s, t) \in[-T, T]^{n} \times$ $[-T, T]^{n}$

$$
\left\|f\left(\cdot+\sum_{i=1}^{n} t_{i} h_{i}\right)-f\left(\cdot+\sum_{i=1}^{n} s_{i} h_{i}\right)\right\|_{1, q} \leqslant C_{1}|t-s|\|f\|_{2, p} .
$$

Now let $f \in H_{0}^{r, p} \backslash\{0\}$. By (3.6), there exists a sequence $\left(f_{n}\right)_{n \in \mathbb{N}} \subset H_{0}^{2, p}$ such that

$$
\sum_{n=1}^{\infty} 2^{(r-1) p n}\left(2^{-n}\left\|f_{n}\right\|_{2, p}+\left\|f-f_{n}\right\|_{1, p}\right)^{p} \leqslant 2^{p}\|f\|_{r, p}^{p} .
$$

Choose the unique $n \in \mathbb{N}$ such that

$$
2^{-n} T<|t-s| \leqslant 2^{-n+1} T
$$

Then by (3.10), (3.11) for some constant $C_{2}=C_{2}(p, q, T)$

$$
\begin{aligned}
\|f(t)-f(s)\|_{1, q} & \leqslant\left\|f(t)-f_{n}(t)\right\|_{1, q}+\left\|f_{n}(t)-f_{n}(s)\right\|_{1, q}+\left\|f_{n}(s)-f(s)\right\|_{1, q} \\
& \leqslant 4 C_{2}\|f\|_{r, p} 2^{-(r-1) n}+\left\|f_{n}\right\|_{2, p} 2^{-n+1} T C_{1} \\
& \leqslant 4 C_{2}\|f\|_{r, p} 2^{-(r-1) n}\left(1+T C_{1}\right) \\
& \leqslant \frac{4 C_{2}\left(1+T C_{1}\right)}{T^{r-1}}|t-s|^{r-1}\|f\|_{r, p}
\end{aligned}
$$

and the assertion is proved. 
Proposition 3.5. Let $T>0, n \in \mathbb{N}, p>1$ and $r \in(1,2]$ such that $r>n p^{-1}+1$. Let $q \in(1, p)$ such that $r>n q^{-1}+1$. Then there exists a constant $C=C(n, p, q, r, T)>0$ such that for any $A \subset W$ we have

$$
C_{1, q}\left(A+M\left(T ; h_{1}, \cdots, h_{n}\right)\right) \leqslant C \cdot C_{r, p}^{\frac{q}{p}}(A)
$$

where

$$
M\left(T ; h_{1}, \cdots, h_{n}\right):=\left\{\sum_{i=1}^{n} s_{i} h_{i},\left|s_{i}\right| \leqslant T\right\}
$$

Proof. Set $s h:=\sum_{i=1}^{n} s_{i} h_{i}$. By changing signs we only need to prove

$$
C_{1, q}\left(\bigcup_{s \in[0, T]^{n}}(A+s h)\right) \leqslant C \cdot C_{r, p}^{\frac{q}{p}}(A) .
$$

Let I denote the set of all rational points in $[0, T]^{n}$. If $O \subset W$ is an open set then so is $\bigcup_{s \in[0, T]^{n}}(O+s h)$ and we have

$$
\bigcup_{s \in[0, T]^{n}}(O+s h)=\bigcup_{s \in I}(O+s h)
$$

Let $e_{O}$ denote the $(r, p)$-equilibrium potential of $O$. Since $e_{O} \geqslant 1_{O}$, we have

$$
\begin{aligned}
C_{1, q}\left(\bigcup_{t \in[0, T]^{n}}(O+t h)\right) & =C_{1, q}\left(\sup _{t \in I} 1_{O}(\cdot+t h)\right) \\
& \leqslant C_{1, q}\left(\sup _{t \in I} e_{O}(\cdot+t h)\right)
\end{aligned}
$$

We set $f(t):=e_{O}(\cdot+t h)$. Applying Lemma 3.4 gives

$$
\|f(t)-f(s)\|_{1, q} \leqslant C\left\|e_{O}\right\|_{r, p}|t-s|^{r-1} .
$$

For $\gamma \in\left[0, r-1-n q^{-1}\right)$, by Theorem 3.2 there exists a $C_{1, q^{-}}$quasicontinuous modification $\left\{\xi_{t}(\cdot), t \in[0, T]^{n}\right\}$ of $\left\{e_{O}(\cdot+t h), t \in[0, T]^{n}\right\}$ such that

$$
C_{1, q}\left[\sup _{s, t \in[0, T]^{n} s \neq t}\left(\frac{\left.\mid \xi_{t}-\xi_{s}\right) \mid}{|t-s|^{\gamma}}\right)\right] \leqslant C\left\|e_{O}\right\|_{r, p}^{q}
$$

for some constant $C=C(n, p, q, r, T)$ which may be different from that in (3.15). In particular, taking $\gamma=0$ we obtain

$$
C_{1, q}\left(\sup _{s \neq t}\left|\xi_{t}-\xi_{s}\right|\right) \leqslant C \cdot\left\|e_{O}\right\|_{r, p}^{q}
$$

Hence

$$
\begin{aligned}
C_{1, q}\left(\sup _{t \in I} e_{O}(\cdot+t h)\right) & =C_{1, q}\left(\sup _{t \in I}\left|\xi_{t}\right|\right) \\
\leqslant & C_{1, q}\left(\sup _{t \in[0, T]}\left|\xi_{t}-\xi_{0}\right|+\left|\xi_{0}\right|\right) \\
\leqslant & (C+1) \cdot\left\|e_{O}\right\|_{r, p}^{q} \\
= & (C+1) \cdot C_{r, p}^{\frac{q}{p}}(O) .
\end{aligned}
$$


Thus (3.13) is proved for open sets. For general $A$, we have for $O \supset A, O$ open

$$
\begin{aligned}
C_{1, q}\left(\bigcup_{s \in[0, T]^{n}}(A+s h)\right) & \leqslant C_{1, q}\left(\bigcup_{s \in[0, T]^{n}}(O+s h)\right) \\
& \leqslant(C+1) \cdot C_{r, p}^{\frac{q}{p}}(O) .
\end{aligned}
$$

Consequently,

$$
\begin{aligned}
C_{1, q}\left(\bigcup_{s \in[0, T]^{n}}(A+s h)\right) & \leqslant(C+1) \cdot \inf \left\{C_{r, p}^{\frac{q}{p}}(O), O \supset A\right\} \\
& =(C+1) \cdot C_{r, p}^{\frac{q}{p}}(A),
\end{aligned}
$$

as desired.

\section{ACKNOWLEDGEMENT}

We would like to thank Terry Jegaraj for spotting a gap in the proof of Lemma 2.1 in the first version of this paper.

Financial support of the BiBoS research center and the DFG through the Research Group "Spectral analysis, asymptotic distributions and stochastic dynamics", as well as the DFG and the MPG through the project "SPDE's with non-Gaussian white noise" is gratefully acknowledged.

\section{REFERENCES}

[1] Albeverio, S., Kondratiev, Y. G. and Röckner, M. Ergodicity for the stochastic dynamics of quasiinvariant measures with applications to Gibbs states. J. Funct. Anal. 149 (1997), no. 2, 415-469.

[2] Albeverio, S. and Röckner, M.: Classical Dirichlet forms on topological vector spaces - closability and a Cameron-Martin formula. J. Funct. Anal. 88 (1990), no. 2, 395-436.

[3] Bergh, J.; Löfström, J. Interpolation spaces. An introduction. Grundlehren der Mathematischen Wissenschaften, No. 223. Springer-Verlag, Berlin-New York, 1976.

[4] Hu, J. and Ren, J.: Infinite Dimensional Quasi Continuity, Path Continuity and Ray Continuity of Functions with Fractional Regularity. J. Math. Pures Appl. 80,1(2001), pp.131-152.

[5] Ma, Z-M. and Röckner, M. Introduction to the theory of (nonsymmetric) Dirichlet forms. Universitext. Springer-Verlag, Berlin, 1992

[6] Ren, J. and Röckner, M.: Ray Hölder-continuity for fractional Sobolev spaces in infinite dimensions and applications, Prob. Theory and Rel. Fields, 117 (2000), n.2, 201-220.

[7] Röckner, M. and Schied, A.: Rademacher's theorem on configuration spaces and applications. $J$. Funct. Anal. 169 (1999), no. 2, 325-356.

[8] Shigekawa, I.: Sobolev spaces of Banach-valued functions associated with a Markov process, Probab. Th. Relat. Fields, 99 (1994), pp. 425-441.

[9] Triebel, H.: Interpolation Theory, Function Spaces, Differential Operators, North-Holland Publishing Company, Amsterdam-New York-Oxford, 1978. 